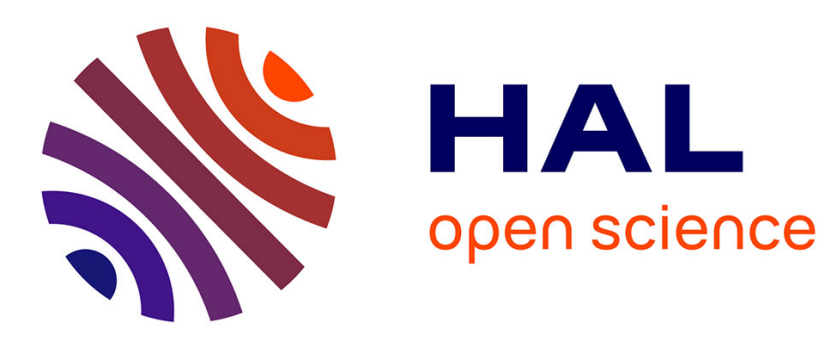

\title{
Biomarkers of vascular dysfunction and cognitive decline in patients with Alzheimer's disease: no evidence for association in elderly subjects
}

Alice Breining, Jean-Sébastien Silvestre, Bénédicte Dieudonné, José Vilar, Véronique Faucounau, Marc Verny, Christian Néri, Chantal M. Boulanger, Jacques Boddaert

\section{To cite this version:}

Alice Breining, Jean-Sébastien Silvestre, Bénédicte Dieudonné, José Vilar, Véronique Faucounau, et al.. Biomarkers of vascular dysfunction and cognitive decline in patients with Alzheimer's disease: no evidence for association in elderly subjects. Aging Clinical and Experimental Research, 2016, pp.1-9. 10.1007/s40520-016-0535-4 . hal-01383389

\section{HAL Id: hal-01383389 \\ https://hal.sorbonne-universite.fr/hal-01383389}

Submitted on 18 Oct 2016

HAL is a multi-disciplinary open access archive for the deposit and dissemination of scientific research documents, whether they are published or not. The documents may come from teaching and research institutions in France or abroad, or from public or private research centers.
L'archive ouverte pluridisciplinaire HAL, est destinée au dépôt et à la diffusion de documents scientifiques de niveau recherche, publiés ou non, émanant des établissements d'enseignement et de recherche français ou étrangers, des laboratoires publics ou privés. 


\section{Biomarkers of vascular dysfunction and cognitive decline in patients with Alzheimer's disease: no evidence for association in elderly subjects}

Alice Breining ${ }^{1,2}$, MD, Jean-Sébastien Silvestre ${ }^{3}$, Bénédicte Dieudonné ${ }^{1}$, José Vilar $^{3}$, Véronique Faucounau ${ }^{1}$, MD, Marc Verny ${ }^{1,2}$, MD,PhD, Christian Néri ${ }^{2}$, Chantal M. Boulanger $^{3}$, Jacques Boddaert ${ }^{1,2}, \mathrm{MD}, \mathrm{PhD}$

(1) APHP, DHU FAST, Groupe Hospitalier Pitié Salpêtrière - Charles Foix, Centre de Gériatrie, F-75013, Paris, France

(2) Sorbonne Universités, UPMC Univ Paris 06, CNRS, UMR 8256 B2A , F-75005, Paris, France

(3) INSERM, U970, Paris Cardiovascular Research Center-PARCC; and Université Paris Descartes, Sorbonne Paris Cité, UMR-S970, Paris, France

\section{Corresponding author :}

Dr Alice Breining

Centre de Gériatrie

Groupe Hospitalier Pitié-Salpêtrière

47-83, Bd de l'Hôpital

75651 Paris Cedex 13

France

tel: 33142164117 ; fax: 33142160325

e-mail: alice.breining@psl.aphp.fr

Conflict of Interest and Sources of Funding : none declared 


\section{ABSTRACT}

Background: Several studies have suggested that vascular dysfunction plays an important role in Alzheimer's disease.

Aims: We hypothesized that significant differences might be observed in the levels of blood endothelial biomarkers across elderly population of subjects with dementia.

Methods: We analyzed, in a prospective monocentric study, 3 different endothelial biomarkers, endothelial microparticles (EMP), endothelial progenitor cells (EPC) and circulating endothelial cells (CEC) in 132 older patients who underwent a full evaluation of a memory complaint.

Results: There was no difference in specific EMP, EPC or CEC levels between demented or non demented patients, nor considering cognitive decline.

Discussion: Blood endothelial biomarkers may be too sensitive and it is likely that the multimorbidity observed in our patients may lead to opposite and confounding effects on endothelial biomarkers levels.

Conclusion: Unlike younger AD patients, our results suggest that endothelial biomarkers are not valuable for the diagnosis of dementia in elderly patients.

Key words : endothelial microparticles, circulating endothelial cells, endothelial progenitors cells, dementia, older population 


\section{INTRODUCTION}

Several studies have suggested that vascular dysfunction plays important roles in Alzheimer's disease (AD). Current evidence suggests a decreased vascular density in ageing, leukoaraiosis and AD. Importantly, cerebrovascular dysfunction may precede cognitive dysfunction and neurodegeneration [1]. A role for cerebrovascular dysfunction has also been suggested by epidemiological studies and associations between $\mathrm{AD}$ and atherosclerosis [2] as well as between $\mathrm{AD}$ and cerebrovascular disease [3, 4]. Additionally, the majority of communitydwelling older persons with dementia often have multiple brain pathologies, underscored by $\mathrm{AD}$ and vascular diseases [5]. Finally, people who suffer from both diseases, AD and infarctions, have a more severe cognitive impairment than those suffering from only one disease $[6,7]$, suggesting that neurodegeneration and cerebrovascular disease may be tightly associated in $\mathrm{AD}$.

Endothelial microparticles (EMP), endothelial progenitor cells (EPC) and circulating endothelial cells (CEC) have emerged as useful markers to evaluate cardiovascular event risk and pathology prognosis. Microparticles (MP) are submicron membrane vesicles expressing a panel of oxidized phospholipids and proteins specific of the cells from which they originate. Increase of MP levels in plasma, particularly those of endothelial origin, reflects cellular injury and have been proposed as surrogate marker of vascular dysfunction [8, 9]. CEC are positive for CD 146 and their detection in a healthy adult is a rare event. In vitro, CEC inhibit EPC proliferation and migration, thus impacting on the regenerative capacity of vessels [10]. High levels of CEC are associated with the development of various vascular diseases [11, 12]. EPC can derive from bone marrow and participate to the maintenance of the endothelium by replacing dysfunctional endothelial cells or releasing pro-angiogenic growth factors. Interestingly, the number and function of EPC inversely correlate with the risk of cardiovascular disease [13]. 
Although EMP, EPC and CEC measures have clinical potential to assess vascular dysfunction, a limited number of studies have analysed the diagnostic and prognostic value of these candidate biomarkers in patients with cognitive impairment. An association between EPC and the Mini-Mental State Examination score has been suggested in AD but not in non AD neurodegenerative disease $[14,15]$. Some circulating EMP subpopulations might also be associated with cognitive decline in AD patients [16]. Here, we evaluated the diagnostic and prognosis value of endothelial dysfunction biomarkers in 132 community dwelling older persons who present a memory complaint. To fully assess the putative vascular dysfunction in these patient populations, we analyzed, at the same time, EMP, EPC and CEC levels.

\section{PATIENTS AND METHODS}

\section{Setting}

This observational study was performed during an 8-month period from November 1, 2010 through June 30, 2011 and was designed to analyze endothelial biomarkers in patients with memory complaint. This study took place in a Memory Center of the Geriatric Department of Hospital Pitié-Salpètrière, Paris, France. This study has been approved by the ethical committee (CPP Pitié-Salpêtrière, Ile-de-France VI, Paris, France).

\section{Patients}

During the period study, community dwelling older patients consulting for a memory complaint were prospectively included. No blood sample was collected specifically for the study. Patients were excluded if they presented with cancer or any acute medical condition that could influence endothelial biomarkers, such as acute coronary syndrome, acute ischemic stroke, and infection. A full evaluation was made for each patient, including medical history, complete physical examination, exhaustive biology, neuropsychological assessment and 
cerebral imaging. If needed, they also had a lumbar punction and/or cerebral scintigraphy. Cardiovascular history was established from medical records.

Diagnosis of cognitive impairment was determined by a multidisciplinary staff and patients were classified into three groups: mild cognitive impairment (MCI) group, dementia group and control group without MCI or dementia (see supplementary methodology). A rapid cognitive decline (RCD) was defined as a decrease of the Mini-Mental State Examination (MMSE) score of 6 points per year [17].

Patients with dementia were followed at least one year after cognitive evaluation. Surviving patients and their family or caregivers were seen for a physical examination with a MMS score according to our routine procedure of follow-up. Missing patients were tracked through health care providers, particularly general practitioners, or any acquaintances identified.

\section{Diagnostic criteria for dementia}

Dementia and MCI were diagnosed according to the Diagnostic and Statistical Manual of Mental Disorders, 4th Edition (American Psychiatric Association). AD was diagnosed according to the National Institute of Neurological and Communicative Disorders and Stroke and the Alzheimer's Disease and Related Disorders Association criteria [18], dementia with Lewy Body (DLB) according to the McKeith et al criteria [19], fronto temporal dementia according to the Lund-Manchester criteria [20], vascular dementia according to the National Institute of Neurological Disorders and Stroke and Association Internationale pour la Recherche et l'Enseignement en Neurosciences criteria [21].

\section{Endothelial microparticles isolation and analysis}

As previously described by Amabile et al., MP were quantified in Platelet-free plasma which was obtained by successive centrifugation ( $500 \mathrm{~g}$ for $15 \mathrm{~min}$ followed by 10,000g for $5 \mathrm{~min}$, at room temperature) of $4 \mathrm{ml}$ of citrated blood. Investigators blinded to subject status characterized MPs on a Guava Express Pro® ver. 8.1.1 flow cytometer. Various types of 
EMP were analyzed: CD62e (E-selectin)-positive EMP, CD144 (VE-cadherin)-positive EMP, and whole pool of MP using calcium-dependent-annexin V labelling. We did not need to exclude specific platelet phenotype as CD144 and CD62E are not expressed by platelets. Circulating MP levels were expressed as events per $\mathrm{mL}(\mathrm{ev} / \mathrm{mL})$.

\section{Assays for circulating endothelial cells}

The CEC were counted in whole blood using immunomagnetic separation. Ferrous beads bound to an anti-CD146 monoclonal antibody were mixed with venous blood in a head-overhead mixer for $30 \mathrm{~min}$ at $4^{\circ} \mathrm{C}$. The unbound cells were washed away with buffer. Cells were labelled with orange acridine and counted in a glass counting chamber under a fluorescent microscope. CEC levels were expressed as CEC count/ml. Healthy controls display around 0$10 \mathrm{CEC} / \mathrm{ml}$.

\section{Endothelial progenitors cells}

EPC were also analysed in whole blood sample. Mononuclear cells were isolated with the use of a Ficoll density gradient according to standard protocols and were suspended in $500 \mu \mathrm{l}$ of a phosphate-buffered saline. Immunofluorescent cell staining was performed with the use of the fluorescent conjugated antibody CD34-fluorescein isothiocyanate, KDR (kinase insert domain receptor)-allophycocyanin, and CD133-phycoerythrin. Cell fluorescence was measured immediately after staining, and data were analyzed with the use of FlowJo software (LSRII, Becton Dikinson). Units of all measured components are absolute cell counts obtained after the measurement of 100,000 events. We measured the number of CD34+ cells, the number of CD34+ CD133+ KDR cells, which represents a subpopulation of immature EPC and the number of CD34+ CD133+CD45- cells to exclude cells with inflammatory phenotypes.

\section{Diagnosis and prognosis of biomarkers}


For each patient, endothelial dysfunction biomarkers (EMP, EPC and CEC) were measured (see supplementary methodology). Results were compared according to the result of the cognitive and geriatric evaluation, between patients with dementia, patients with MCI, or patients without any dementia or MCI. Moreover, results were compared according to the type of dementia, considering neurodegenerative, vascular and mixt origin, i.e. neurodegenerative and vascular origin. The prognostic value of endothelial biomarkers was studied with respect to one year mortality. For patients with dementia alive at one year, prognostic value of biomarkers was analyzed according to the existence of RCD cognitive decline or not.

\section{Statistics}

The statistical analysis was performed with the statistical software Xlstat. Qualitative variables are expressed as number and percentage, and the quantitative variables as median and inter quartile rate (IQR). Kruskal-Wallis one-way analysis of variance on ranks and Mann Whitney Test were used for group comparison for quantitative variables. $\chi^{2}$ test was performed for comparisons between groups for categorical variables. A multiple regression analysis (ANCOVA) was performed to identify factors that were independently associated with MP counts. The alpha error was set at 0.05 .

\section{RESULTS}

Among the 373 patients admitted for memory complaint during the study period, 132 patients had a blood sample drawn at hospital, including 82 patients with dementia, 12 with MCI and 38 controls. Among the 82 patients with dementia, 48 individuals (59\%) were diagnosed having $\mathrm{AD}$ (Figure). Among the 38 control patients, 20 individuals (53\%) had depression symptoms. Main characteristics are presented in Table 1 . Mean age of patients was $83 \pm 6$ years old. Patients with dementia had a lower MMSE score $(p<0.0001)$ and a lower IADL 
score $(\mathrm{p}=0.0002)$ compared to MCI and control patients. There were no differences for type and number of cardiovascular risk factors, and associated comorbidities between groups (Table 1). All patients had EMP measurements. Due to technical issues, only 86 patients had CEC measurements and 85 patients EPC measurements. All biomarkers were detected in patients, and we found no significant difference between control patients, patients with MCI and with dementia (table 2). There was no difference between control patients, patients with neurodegenerative disease and patients with vascular and mixed dementia (table 2). All these results were not modified after adjusting for cardiovascular risk factors and comorbidities. In a multiple regression analysis, we observed a correlation between hypertension and high levels of EMP ( $p<0.01$ ), but no correlation was found between EMP and other risk factors.

\section{Long term outcome}

One-year cognitive follow-up was studied. Ten out of 59 living patients with dementia and cognitive follow up had a RCD and were compared to the 49 patients without RCD. There was no significant difference between groups for cardiovascular risk factors and associated comorbidities. Patients with RCD had a lower MMSE at one year $(\mathrm{p}=0.008)$ (Table 3). Patients with RCD had lower annexin V- MP levels compared to patients not having RCD (268/ml [187-401] versus 530/ml [246-1212], $\mathrm{p}=0.04)$. This result was not confirmed with specific EMP. Biomarkers for EPC and CEC did not show any significant towards association (Table 3).

\section{DISCUSSION}

To our knowledge, this is the first study analysing the diagnostic and prognostic value of three different and complementary biomarkers of endothelial dysfunction in older patients with a memory complaint. Altogether, our results suggest that endothelial biomarkers are not valuable neither for the diagnosis of dementia in older patients, whatever the underlying mechanism, nor to 
predict cognitive decline in older patients with dementia.

Those three biomarkers were chosen because they are associated with pathways that regulate endothelial function. Since alteration of vascular homeostasis has been involved in the development of $\mathrm{AD}$, or appears as a key process in neurodegenerative dementia, we hypothesized that significant differences might be observed in the levels of endothelial biomarkers across heterogeneous populations of subjects with dementia. We did not observe any difference between patients with dementia or not, nor between patients with neurodegenerative and vascular or mixed dementias. But we obtain suggestive evidence for differences within the group of demented patients according to their rate of cognitive decline: comparison of biomarkers of endothelial dysfunction between patients with RCD or not revealed a significant difference of Annexin V-positive MPs (Table 3). Since Annexin V labels MP are irrespective of their cellular origin, these results suggest that total MP levels may be associated with the rate of cognitive decline in patients with dementia. But this difference was not observed when specific EMP markers were used.

Previous data showed evidence for an increase of certain circulating EMP phenotypes (CD31+ CD42- and CD62e+ CD42-) correlated with a cognitive decline of AD patients [16]. In this study Xue et al. correlated EMP counts and AD cognitive decline in a multivariate regression analysis including clinical parameters correlated with EMP counts (i.e. age and cholesterol levels). But they didn't investigate correlation between EMP counts and hypertension, nor diabetes and stroke. Yet it is now well demonstrated that hypertension, diabetes and stroke increases EMP counts [8].

Recent studies reported that patients with AD have reduced EPC levels and functions $[14,15$, 22], suggesting that an abnormal capacity to regenerate endothelium is associated with AD. As Kong et al. and Lee et al., we did not found a correlation between CD34+ EPC counts and 
dementia. But Kong et al. assessed that patients with $\mathrm{AD}$ and vascular dementia had lower CD34+CD133+ EPCs counts than controls. Lee et al. found that AD patients have reduced EPC but for instance, Lee et al. cultured and counted EPC from peripheral blood samples [14, 22] whereas in our study, EPC were directly measured in whole blood sample.

Our results do not confirm Kong et al. and Lee et al. results. However, mean age of patients enrolled in our study was higher than those from Lee et al. and Kong et al. ( $83 \pm 7$ years old versus $69.1 \pm 8.3$ years old and $71.4 \pm 2.3$ ). Hence, we cannot exclude that differences in endothelial dysfunction markers in older demented patients could have be influenced by age and numerous comorbidities observed in these patient population.

Indeed, atherosclerosis, artery coronary disease, severe hypertension, metabolic syndrome, diabetes, smoking, chronic renal failure, inflammatory diseases and cancer [8] are known to raise EMP count. Aging, acute coronary syndromes, heart failure are known to decrease EPC level [23]. Likewise, acute coronary syndromes, heart failure, inflammatory diseases and cancer raise CEC level in peripheral blood [24]. In our study, patients had on average 2.9 cardiovascular risk factor and other comorbidities that could influence endothelial biomarker's count. Even though we have excluded patients with acute disease and adjusted our results with cardio-vascular risk factors, it is likely that the multimorbidity observed in our patients may lead to opposite and confounding effects on endothelial biomarkers levels.

Our results do not suggest a correlation between vascular function and AD. Many reasons can explain this result. First, initiation of AD is not a vascular event. Hypoxia resulting from the vascular insufficiency could promote $\beta$ amyloid accumulation and the attendant deleterious effects on the brain. Conversely, amyloid precursor protein could induce vascular damage and worsen vascular insufficiency, which would also lead to brain dysfunction [25]. Synergic or additive interactions between those two pathways may participate to their pathogenic effects. 
A second hypothesis is that vascular dysfunction is substantial at the early stages of the disease but afterward other mechanism may have taken over. In this view, patients included in the study and diagnosed as AD patients are no longer at subclinical stages of the disease. Initiation of $\mathrm{AD}$ may not be a vascular event or vascular dysfunction may be substantial at the early stages of the disease but afterward other mechanism may have taken over. In this view, patients included in the study and diagnosed as AD patients are no longer at subclinical stages of the disease.

The present study has several limitations. First all patients with a memory complaint had an exhaustive biology but only $1 / 3$ had a blood sample taken at hospital and endothelial biomarkers measured. The number of patients included in the study is though limited and measurements of the three endothelial biomarkers are not always available for all the patients for technical reasons. Finally, the definition of RCD is based on patients with AD [17], and this definition may not be relevant for all types of dementia.

\section{CONCLUSION}

Unlike datas on younger $\mathrm{AD}$ patients, our results suggest that endothelial biomarkers are not valuable for the diagnosis of dementia in older patients, whatever the underlying mechanism. Vascular endothelial biomarkers may be too sensitive and not specific enough in older population. This observation, together with previously reported trends, calls for additional studies of the potential of endothelial biomarkers. 
Acknowledgments: The authors thank the INSERM U970 team of the Paris Cardiovascular Research Center for their support.

Declaration of Sources of Funding: none

Conflict of Interests: The authors declare that there is no conflict of interests regarding the publication of this paper.

All authors have read the paper and have agreed to be listed as authors. 


\section{REFERENCES}

1. Brown WR, Thore CR. Review: cerebral microvascular pathology in ageing and neurodegeneration. Neuropathol Appl Neurobiol. 2011;37(1):56-74.

2. Roher AE, Esh C, Rahman A, Kokjohn TA, Beach TG. Atherosclerosis of cerebral arteries in Alzheimer disease. Stroke. 2004;35(1):2623-7.

3. Gorelick PB. Risk factors for vascular dementia and Alzheimer disease. Stroke. 2004;35(1):2620-2.

4. De la Torre JC. Is Alzheimer's disease a neurodegenerative or a vascular disorder? Data, dogma, and dialectics. Lancet Neurol. 2004;3(3):184-90.

5. Schneider JA, Arvanitakis Z, Bang W, Bennett DA. Mixed brain pathologies account for most dementia cases in community-dwelling older persons. Neurology. 2007;69(24):2197-204.

6. Riekse RG, Leverenz JB, McCormick W, Bowen JD, Teri L, Nochlin D, et al. Effect of vascular lesions on cognition in Alzheimer's disease: a community-based study. J Am Geriatr Soc. 2004;52(9):1442-8.

7. Rockwood K, Davis H, MacKnight C, Vandorpe R, Gauthier S, Guzman A, et al. The Consortium to Investigate Vascular Impairment of Cognition: methods and first findings. Can J Neurol Sci. 2003;30(3):237-43.

8. Boulanger C-M, Leroyer A-S, Amabile N, Tedgui A. Circulating endothelial microparticles: a new marker of vascular injury. Ann Cardiol Angeiol (Paris). 2008;57(3):149-54.

9. Nozaki T, Sugiyama S, Koga H, Sugamura K, Ohba K, Matsuzawa Y, et al. Significance of a multiple biomarkers strategy including endothelial dysfunction to improve risk stratification for cardiovascular events in patients at high risk for coronary heart disease. J Am Coll Cardiol. 2009;54(7):601-8.

10. Holmén C, Elsheikh E, Stenvinkel P, Qureshi AR, Pettersson E, Jalkanen S, et al. Circulating inflammatory endothelial cells contribute to endothelial progenitor cell dysfunction in patients with vasculitis and kidney involvement. J Am Soc Nephrol. 2005;16(10):3110-20. 
11. Werner N, Kosiol S, Schiegl T, Ahlers P, Walenta K, Link A, et al. Circulating endothelial progenitor cells and cardiovascular outcomes. N Engl J Med. 2005;353(10):999-1007.

12. Hill JM, Zalos G, Halcox JPJ, Schenke WH, Waclawiw MA, Quyyumi AA, et al. Circulating endothelial progenitor cells, vascular function, and cardiovascular risk. N Engl J Med. 2003;348(7):593-600.

13. Vasa M, Fichtlscherer S, Aicher A, Adler K, Urbich C, Martin H, et al. Number and migratory activity of circulating endothelial progenitor cells inversely correlate with risk factors for coronary artery disease. Circ Res. 2001;89(1):E1-7.

14. Lee S-T, Chu K, Jung K-H, Park H-K, Kim D-H, Bahn J-J, et al. Reduced circulating angiogenic cells in Alzheimer disease. Neurology. 2009;72(21):1858-63.

15. Kong X, Zhang Y, Liu L, Sun N, Zhang M, Zhang J. Endothelial progenitor cells with Alzheimer's disease. Chin Med J. 2011;124(6):901-6.

16. Xue S, Cai X, Li W, Zhang Z, Dong W, Hui G. Elevated plasma endothelial microparticles in Alzheimer's disease. Dement Geriatr Cogn Disord. 2012;34(3-4):174-80.

17. Schmidt C, Wolff M, Weitz M, Bartlau T, Korth C, Zerr I. Rapidly progressive Alzheimer disease. Arch Neurol. 2011;68(9):1124-30.

18. McKhann G, Drachman D, Folstein M, Katzman R, Price D, Stadlan EM. Clinical diagnosis of Alzheimer's disease: report of the NINCDS-ADRDA Work Group under the auspices of Department of Health and Human Services Task Force on Alzheimer's Disease. Neurology. 1984;34(7):939-44.

19. McKeith IG, Galasko D, Kosaka K, Perry EK, Dickson DW, Hansen LA, et al. Consensus guidelines for the clinical and pathologic diagnosis of dementia with Lewy bodies (DLB): report of the consortium on DLB international workshop. Neurology. 1996;47(5):1113-24.

20. Clinical and neuropathological criteria for frontotemporal dementia. The Lund and Manchester Groups. J Neurol Neurosurg Psychiatr. 1994;57(4):416-8. 
21. Román GC, Tatemichi TK, Erkinjuntti T, Cummings JL, Masdeu JC, Garcia JH, et al. Vascular dementia: diagnostic criteria for research studies. Report of the NINDS-AIREN International Workshop. Neurology. 1993;43(2):250-60.

22. Lee S-T, Chu K, Jung K-H, Jeon D, Bahn J-J, Kim J-H, et al. Dysfunctional characteristics of circulating angiogenic cells in Alzheimer's disease. J Alzheimers Dis. 2010;19(4):1231-40.

23. Lee PSS, Poh KK. Endothelial progenitor cells in cardiovascular diseases. World J Stem Cells. 2014;6(3):355-66.

24. Erdbruegger U, Haubitz M, Woywodt A. Circulating endothelial cells: a novel marker of endothelial damage. Clin Chim Acta. 2006;373(1-2):17-26.

25. Iadecola $\mathrm{C}$. The overlap between neurodegenerative and vascular factors in the pathogenesis of dementia. Acta Neuropathol. 2010;120(3):287-96. 


\section{TABLES}

Table 1. Baseline characteristics of patients.

\begin{tabular}{|lcccc|}
\hline & $\begin{array}{c}\text { Control patients } \\
\mathbf{n = 3 8}\end{array}$ & $\begin{array}{c}\text { MCI } \\
\mathbf{n = 1 2}\end{array}$ & $\begin{array}{c}\text { Dementia } \\
\mathbf{n = 8 2}\end{array}$ & p-value \\
\hline Age (years) & $80.7 \pm 5.2$ & $83.5 \pm 6.1$ & $83.2 \pm 6.4$ & NS \\
Gender (female) & $27 \pm 71.1$ & $4 \pm 33.3$ & $49 \pm 59.8$ & NS \\
MMSE score & $25.6 \pm 3.2$ & $26.4 \pm 2.8$ & $21.7 \pm 4.6$ & $<0.0001$ \\
IADL score & $9.8 \pm 4.0$ & $12.1 \pm 2.9$ & $7.8 \pm 3.7$ & 0.0002 \\
\hline Cardiovascular risk factors, $\boldsymbol{n}(\boldsymbol{\%})$ & & & \\
Hypertension & $23(60.5)$ & $6(50)$ & $55(67.1)$ & NS \\
Dyslipidaemia & $15(39.5)$ & $5(41.7)$ & $25(30.5)$ & NS \\
Diabetes mellitus & $9(23.7)$ & $3(25)$ & $13(15.9)$ & NS \\
Obesity & $12(31.6)$ & $4(33.3)$ & $18(22)$ & NS \\
Smoking & $1(2.6)$ & $3(25)$ & $10(12.3)$ & NS \\
\hline Cardiovascular risk factors Total, $\boldsymbol{n}(\boldsymbol{\%})$ & & & \\
1 & $5(13.2)$ & 0 & $8(9.8)$ & NS \\
2 & $12(31.6)$ & $4(33.3)$ & $31(37.8)$ & NS \\
3 & $9(23.7)$ & $3(25)$ & $21(25.6)$ & NS \\
4 & $7(18.4)$ & $2(16.7)$ & $10(12.2)$ & NS \\
5 & $5(13.2)$ & $2(16.7)$ & $9(11)$ & NS \\
6 & 0 & $1(8.3)$ & $3(3.7)$ & NS \\
\hline Comorbidities, $\boldsymbol{n}(\%)$ & & & & \\
Atrial fibrillation & $5(13.2)$ & $2(16.7)$ & $18(22)$ & NS \\
Stroke & $2(5.3)$ & $1(8.3)$ & $9(11)$ & NS \\
Coronary Heart Disease & $3(7.9)$ & $1(8.3)$ & $10(12.2)$ & NS \\
Arteritis & $2(5.3)$ & $1(8.3)$ & $6(7.3)$ & NS \\
Heart Failure & $1(2.6)$ & 0 & $3(3.7)$ & NS \\
\hline
\end{tabular}

Results were expressed as mean \pm sd, number of patients (percentages). MMSE: Mini-

mental State Examination; IADL: Instrumental Activities of Daily Living; MCI: Mild

Cognitive Impairment. 
Table 2: Comparison of biomarkers of endothelial dysfunction between control patients, patients with MCI, dementia and according to the type of dementia.

\begin{tabular}{|c|c|c|c|c|c|c|}
\hline & Control & MCI & $\begin{array}{c}\text { Dementia } \\
\text { All }\end{array}$ & $\begin{array}{c}\text { Neurodegenerative } \\
\text { Dementia }\end{array}$ & $\begin{array}{l}\text { Vascular } \\
\text { Dementia }\end{array}$ & \\
\hline Microparticules & $(n=38)$ & $(n=12)$ & $(n=82)$ & $(n=63)$ & $(n=19)$ & NS \\
\hline \multirow{2}{*}{ Annexin V+ MP } & 416 & 386 & 549.7 & 531 & 688 & NS \\
\hline & [149-1020] & [170-1093] & [248-1110] & [233-1063] & [380-1255] & \\
\hline \multirow[t]{2}{*}{ CD144+ EMP } & 64 & 92 & 84.9 & 81 & 100 & NS \\
\hline & [46-143] & [37-123] & [39-174] & [39-151] & [39-210] & \\
\hline \multirow[t]{2}{*}{ CD62E+ EMP } & 78 & 63 & 92 & 94 & 92 & NS \\
\hline & [39-106] & [37-109] & [54-118] & [56-117] & [39-166] & \\
\hline \multirow[t]{3}{*}{ CEC } & $(n=25)$ & $(\mathrm{n}=11)$ & $(n=50)$ & $(n=40)$ & $(n=10)$ & \\
\hline & 6 & 6 & 13 & 15 & 3 & NS \\
\hline & {$[2-72]$} & {$[2-16]$} & {$[2-44]$} & {$[4.8-47.0]$} & {$[0.5-18]$} & \\
\hline EPC & $(n=25)$ & $(n=10)$ & $(n=49)$ & $(n=39)$ & $(n=10)$ & \\
\hline \multirow{2}{*}{ CD34+ } & 0.07 & 0.11 & 0.07 & 0.07 & 0.09 & NS \\
\hline & {$[0.06-0.08]$} & {$[0.07-0.17]$} & {$[0.05-0.10]$} & {$[0.05-0.10]$} & {$[0.05-0.13]$} & \\
\hline \multirow[t]{2}{*}{ CD34+ CD133+ VEGF+ } & 0.3 & 1.2 & 0.4 & 0.5 & 0.2 & NS \\
\hline & {$[0.0-0.7]$} & {$[0-8.5]$} & {$[0.0-1.4]$} & [0.06-1.4] & {$[0.03-1.1]$} & \\
\hline \multirow[t]{2}{*}{ CD34+ CD133+ CD45- } & 57 & 51 & 57 & 56.6 & 58.2 & NS \\
\hline & [52-61] & [41-61] & [46-64] & [43.9-64.9] & [53.0-63.9] & \\
\hline
\end{tabular}

Results are expressed as median values and ranges. Circulating MP levels were expressed as events per $\mathrm{mL}$, CEC levels as CEC count/ml and EPC levels as absolute cell counts obtained after the measurement of 100,000 events. EMP: Endothelial Microparticles, CEC: circulating endothelial cells, EPC: endothelial progenitors cells, MCI: Mild Cognitive Impairment. 
Table 3. Characteristics of patients and comparison of three biomarkers of endothelial dysfunction according to cognitive decline during follow-up.

\begin{tabular}{|c|c|c|c|}
\hline & $\begin{array}{c}\text { RCD } \\
(\mathrm{n}=10)\end{array}$ & $\begin{array}{c}\text { No RCD } \\
(n=49)\end{array}$ & p-value \\
\hline Age (years), mean \pm sd & $81,3 \pm 5,3$ & $81,4 \pm 5,2$ & NS \\
\hline Gender (female), n (\%) & $8(80 \%)$ & $28(57 \%)$ & NS \\
\hline MMSE score, mean \pm sd & $24 \pm 3.2$ & $21.5 \pm 6$ & NS \\
\hline $\mathrm{MMS}+1$ score, mean $\pm \mathrm{sd}$ & $16.4 \pm 4$ & $20.1 \pm 5.3$ & 0.008 \\
\hline IADL mean \pm sd & $8 \pm 4.5$ & $8.8 \pm 3.3$ & NS \\
\hline \multicolumn{4}{|c|}{ Cardiovascular risk factors, $n(\%)$} \\
\hline Hypertension & $6(60 \%)$ & $32(65 \%)$ & NS \\
\hline Dyslipidaemia & $3(30 \%)$ & $16(33 \%)$ & NS \\
\hline Diabetes mellitus & $2(20 \%)$ & $10(20 \%)$ & NS \\
\hline Obesity & $2(20 \%)$ & $16(32 \%)$ & NS \\
\hline Smoking & $1(10 \%)$ & $4(8 \%)$ & NS \\
\hline \multicolumn{4}{|c|}{ Cardiovascular risk factors Total, $\boldsymbol{n}(\%)$} \\
\hline 1 & $1(10 \%)$ & $3(6 \%)$ & NS \\
\hline 2 & $5(50 \%)$ & $18(37 \%)$ & NS \\
\hline 3 & $2(20 \%)$ & $13(27 \%)$ & NS \\
\hline 4 & $1(10 \%)$ & $8(16 \%)$ & NS \\
\hline 5 & $1(10 \%)$ & $4(18 \%)$ & NS \\
\hline 6 & 0 & $3(6 \%)$ & NS \\
\hline \multicolumn{4}{|l|}{ Comorbidities, $n(\%)$} \\
\hline ACFA & $1(10 \%)$ & $10(20 \%)$ & NS \\
\hline Stroke & 0 & $5(10 \%)$ & NS \\
\hline Coronary & $3(30 \%)$ & $6(12 \%)$ & NS \\
\hline AOMI & 0 & $2(4 \%)$ & NS \\
\hline Heart failure & 0 & $2(4 \%)$ & NS \\
\hline Microparticles & $(n=10)$ & $(n=49)$ & \\
\hline Annexin V+ MP & $286[187-401]$ & $530[246-1212]$ & 0.04 \\
\hline CD144+ EMP & $46[6.6-105]$ & $97[56-179]$ & $\begin{array}{l}\text { No } \\
\text { NS }\end{array}$ \\
\hline CD62E+ EMP & $91[48-107]$ & $90[54-116]$ & NS \\
\hline \multirow[t]{2}{*}{$C E C$} & $(\mathrm{n}=9)$ & $(n=29)$ & \\
\hline & $37[10-101]$ & $12[2-32]$ & NS \\
\hline$E P C$ & $(n=9)$ & $(n=29)$ & \\
\hline CD34+ & $0.07[0.05-0.09]$ & $0.07[0.06-0.09]$ & NS \\
\hline CD34+ CD133+ VEGF+ & $0.1[0-1.7]$ & $0.48[0.0-0.1]$ & NS \\
\hline CD34+ CD133+ CD45- & $55[48-61]$ & $59[44-63]$ & NS \\
\hline
\end{tabular}

Results are expressed as median values and ranges. Circulating MP levels were expressed as events per $\mathrm{mL}$, CEC levels as CEC count/ml and EPC levels as absolute cell counts obtained after the measurement of 100,000 events. EMP: Endothelial Microparticles, CEC: circulating endothelial cells, EPC: endothelial progenitors cells, MCI: Mild Cognitive Impairment, RCD: rapid cognitive decline. MMSE: Mini-mental State Examination, IADL: Instrumental Activities of Daily Living. 


\section{FIGURE}

Figure. Flow chart of community dwelling older persons who present a memory complaint

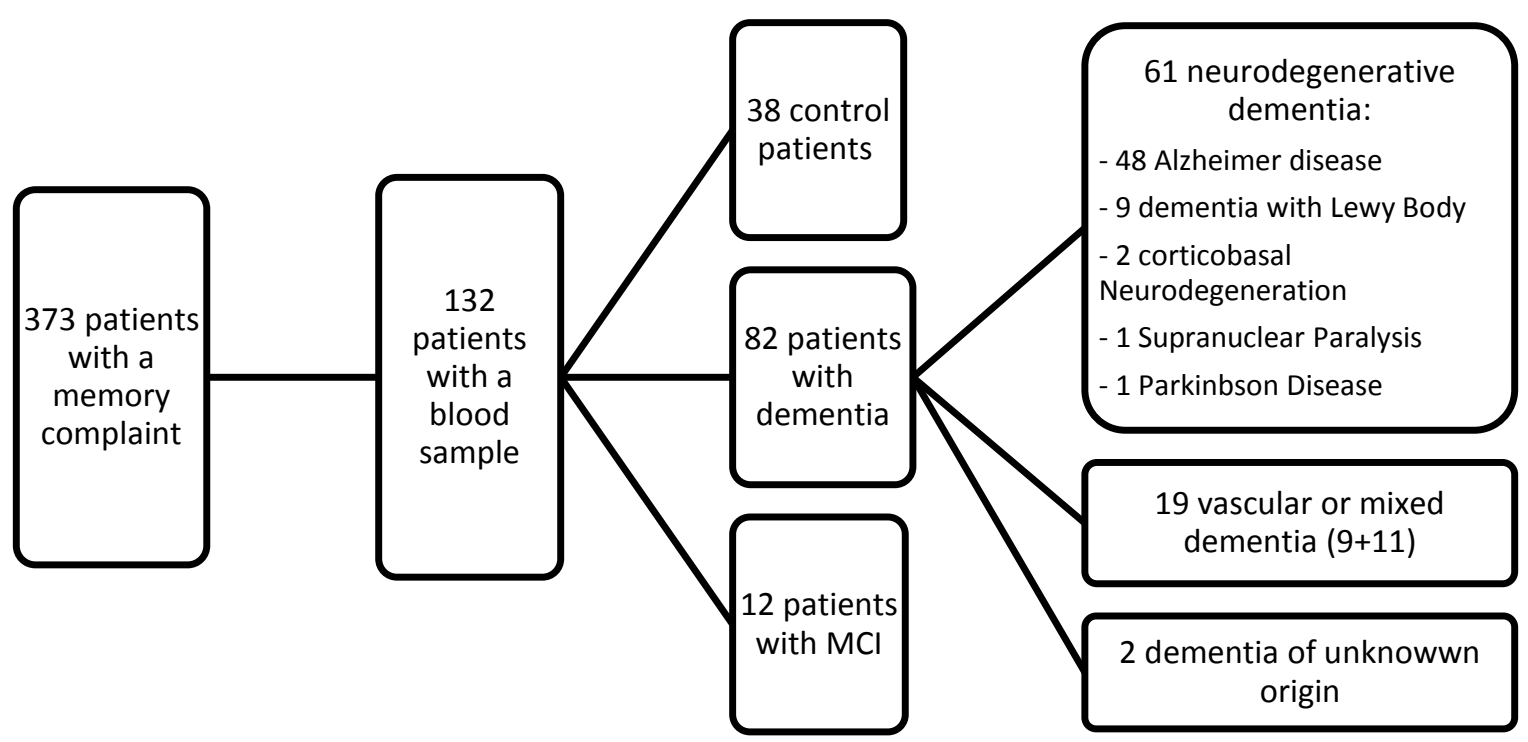

